ARTICLE

DOI: $10.1038 / s 41467-018-06378-9$

\title{
One-step generation of multiple gene knock-outs in the diatom Phaeodactylum tricornutum by DNA-free genome editing
}

\author{
Manuel Serif ${ }^{1,2,3}$, Gwendoline Dubois ${ }^{1,2,3}$, Anne-Laure Finoux ${ }^{1,2,3}$, Marie-Ange Teste ${ }^{1,2,3}$, Denis Jallet ${ }^{1,2,3}$ \& \\ Fayza Daboussi ${ }^{1,2,3}$
}

Recently developed transgenic techniques to explore and exploit the metabolic potential of microalgae present several drawbacks associated with the delivery of exogenous DNA into the cells and its subsequent integration at random sites within the genome. Here, we report a highly efficient multiplex genome-editing method in the diatom Phaeodactylum tricornutum, relying on the biolistic delivery of CRISPR-Cas9 ribonucleoproteins coupled with the identification of two endogenous counter-selectable markers, PtUMPS and PtAPT. First, we demonstrate the functionality of RNP delivery by positively selecting the disruption of each of these genes. Then, we illustrate the potential of the approach for multiplexing by generating double-gene knock-out strains, with $65 \%$ to $100 \%$ efficiency, using RNPs targeting one of these markers and PtAureola, a photoreceptor-encoding gene. Finally, we created triple knock-out strains in one step by delivering six RNP complexes into Phaeodactylum cells. This approach could readily be applied to other hard-to-transfect organisms of biotechnological interest.

\footnotetext{
${ }^{1}$ INSA, UPS, INP, LISBP, Université de Toulouse, 135 Avenue de Rangueil, F-31077 Toulouse, France. ${ }^{2}$ INRA, LISBP, UMR792, 135 Avenue de Rangueil, F31077 Toulouse, France. ${ }^{3}$ CNRS, LISBP, UMR5504, 135 Avenue de Rangueil, F-31077 Toulouse, France. These authors contributed equally: Manuel Serif, Gwendoline Dubois. Correspondence and requests for materials should be addressed to F.D. (email: fayza.daboussi@insa-toulouse.fr)
} 
T he term microalgae encompasses a vast diversity of organisms, which make major contributions to biogeochemical cycles on a global scale ${ }^{1}$. A very attractive aspect concerns the ability of most microalgae to perform photosynthesis, allowing the generation of biological compounds from carbon dioxide $\left(\mathrm{CO}_{2}\right)$ as an inorganic carbon source and light as an energy source. Thus, these versatile cell factories have the potential to become key enablers for industrial biotechnology. Today, only a few microalgae species are commercially exploited ${ }^{2}$. They include the diatom Phaeodactylum tricornutum, which naturally synthesizes numerous marketable compounds ${ }^{3}$, such as pigments and long-chain omega-3 fatty acids ${ }^{4,5}$.

Genetic engineering is a promising approach to improve the metabolic potential of microalgae and obtain further insights into their metabolism and physiology. Custom molecular scissors have recently emerged as useful tools to induce site-specific modifications to the genome of diatoms ${ }^{6-12}$. These nucleases recognize and introduce a double strand break (DSB) at a target genomic sequence, which is repaired by non-homologous end joining (NHEJ) with potentially associated targeted mutagenesis (TM) or by homology-directed repair (HDR) in the presence of a DNA donor template ${ }^{13}$. To date, meganucleases, TALE nucleases, and the CRISPR-Cas9 system have all been successfully applied to inactivate single target genes in Phaeodactylum, either for functional analysis ${ }^{6-12}$ or to redirect natural metabolism towards increased neutral lipid biosynthesis ${ }^{6}$. However, the construction of complex synthetic metabolic pathways requires the simultaneous introduction of multiple genetic modifications, as exemplified by the ten genome changes required to generate a hydrocortisone-producing yeast ${ }^{14}$. Such a challenge has not yet been addressed by the microalgal community.

Indeed, the "nuclease-driven" genetic engineering of diatoms is still in its infancy. Until now, it has been mediated by transforming plasmids encoding a nuclease and an antibiotic resistance cassette into the cells, both then stably integrated at random sites within the nuclear genome ${ }^{6-11}$. Disadvantages of this approach include: the low transformation efficiencies (less than $10^{-6}$ ); the long-term expression of the nuclease can potentially induce offtarget cleavage $^{15}$; the random integration of all or part of the plasmid DNA into the genome can lead to undesired gene disruptions or uncontrolled effects on gene expression near the integration site(s); and the impossibility to eliminate background mutations or integrated transgenes through outcrossing in Phaeodactylum, as it is a diploid organism with no known sexual reproduction. Alternative genome editing methodologies must be developed to circumvent these issues. Recently, an episome-based protocol that avoids genomic integration has been described ${ }^{12}$ that still elicits Cas9 expression over several generations, with potential toxicity or off-target effects ${ }^{15}$. Another possibility is to establish DNA-free genome-editing approaches, as illustrated in only one microalga thus far: the chlorophyte Chlamydomonas reinhardtii ${ }^{16-19}$. In Chlamydomonas, the direct transformation of Cas9/ single-guide RNA (sgRNA) ribonucleoprotein complexes (RNPs) can drive TM, but requires the use of cell-wall less mutants ${ }^{18,19}$ or a plasmid carrying an antibiotic resistance cassette must be cotransformed with the RNP for selection ${ }^{17}$. The challenge in Phaeodactylum rests on both the introduction of RNP complexes through the complex cell wall and the development of an antibiotic-free selection method to enrich for transformants harboring TM events. We thus propose a strategy relying on the simultaneous co-delivery of multiple RNP complexes by biolistic, one targeting an endogenous gene for which inactivation confers positive selection, and the others targeting genes of interest.

Here, we identify two endogenous marker genes in Phaeodactylum, one homologous to $U R A 3^{20}$, the other to $A P T^{21}$. We demonstrate that their inactivation, by either nucleases expressed from the genome or RNP complexes, leads to 5-fluoroorotic acid (5-FOA) and 2-fluoroadenine (2-FA) resistance, a property that can be used for the direct selection of the transformants. Combining RNPs targeting either of these marker genes and another gene of interest, PtAureola, encoding a blue-light photoreceptor/ transcription factor ${ }^{22}$, we generated multiple knock-outs in a single step at high frequency. Our results illustrate that "DNAfree" genome-editing can be achieved in diatoms. We anticipate that it can be transferred to any other hard-to-transfect organism, as it relies on well conserved endogenous selection markers.

\section{Results}

Inactivation of the UMP synthase gene using TALENs. We first evaluated the use of endogenous positive selectable markers for DNA-free genome editing by attempting to produce knock-out (KO) strains of the PtUMPS gene (Phatr3_J11740), the Phaeodactylum URA3 homolog of which knock-down increases 5-FOA tolerance while leading to uracil auxotrophy ${ }^{20}$. We designed a TALEN pair to target the orotidine- 5 '-phosphate decarboxylase domain-encoding sequence within PtUMPS exon 1 (Fig. 1a). The corresponding TALEN-encoding plasmids were assembled and co-delivered by biolistic bombardment into wild-type (WT) Phaeodactylum cells (NCMA strain), together with a plasmid conferring resistance to nourseothricin (NAT). Of the 30 NATresistant colonies that appeared, five showed successful genomic integration of the TALEN monomer genes, as revealed by PCR. These colonies were then spotted onto 5-FOA selective medium supplemented with uracil 20 ; two of five colonies, hereafter referred to as $8 \mathrm{~A} 2$ and $12 \mathrm{~A} 1$, grew in this condition. Genomic PCR amplification of PtUMPS from $8 \mathrm{~A} 2$ and $12 \mathrm{~A} 1$ revealed the presence of mixed cell populations, a phenomenon referred to as mosaicism $^{13}$ (Fig. 1b). Based on chromatogram deconvolutions, the TIDE calculation software ${ }^{23}$ suggested a variety of INDELs at the PtUMPS locus for the 5-FOA resistant colonies (Fig. 1c). Therefore, we subcloned the $8 \mathrm{~A} 2$ and $12 \mathrm{~A} 1$ populations on medium containing 5-FOA and uracil and then sequenced the PtUMPS locus in 36 individual subclones for $8 \mathrm{~A} 2$ and 20 individual subclones for $12 \mathrm{~A} 1$. As expected, all showed mutagenic events (examples are depicted in Fig. 1d). In 19 of $2012 \mathrm{Al}$ subclones, both PtUMPS alleles showed mutagenic events based on polymorphism patterns present upstream and downstream of the target site (Supplementary Fig. 1). Only one allele was amplified in 35 of $368 \mathrm{~A} 2$ subclones and 1 of $2012 \mathrm{~A} 1$ subclones (Fig. 1d), suggesting large insertions or deletions in the other allele ${ }^{6,9}$, a phenomenon known as loss of heterozygosity $(\mathrm{LOH})^{24}$. In the detected allele, INDELs were systematically present.

We further characterized two 8A2 subclones, 8A2_1 and 8A2_8, and two $12 \mathrm{~A} 1$ subclones, $12 \mathrm{~A} 1 \_2$ and $12 \mathrm{~A} 1 \_8$, for their ability to grow in liquid $\mathrm{F} / 2$ medium with or without uracil and with or without 5-FOA (Fig. 1e-g; Supplementary Fig. 2). The WT strain (NCMA) and a colony (2A3) only transformed with the $\mathrm{NAT}^{\mathrm{R}}$ plasmid were used as controls. All subclones carrying mutations in PtUMPS exhibited growth in the presence of 5-FOA and uracil (Fig. 1e), in contrast to the two controls. This confirms the expected phenotype for the PtUMPS KO concerning 5-FOA resistance. The quasi-absence of growth observed for the PtUMPS mutants in the absence of uracil confirmed their auxotrophy for uracil (Fig. 1f). Importantly, their growth kinetics in medium supplemented with uracil was equivalent to that of controls cultivated in the presence or absence of uracil (Fig. 1g). This suggests that the PtUMPS mutation has no impact on cell fitness under our culture conditions, a major prerequisite for further laboratory and industrial applications. We thus confirmed that PtUMPS KO leads to 5-FOA ${ }^{\mathrm{R}}$ and uracil auxotrophy, a phenotype that can be used for direct selection. 
a
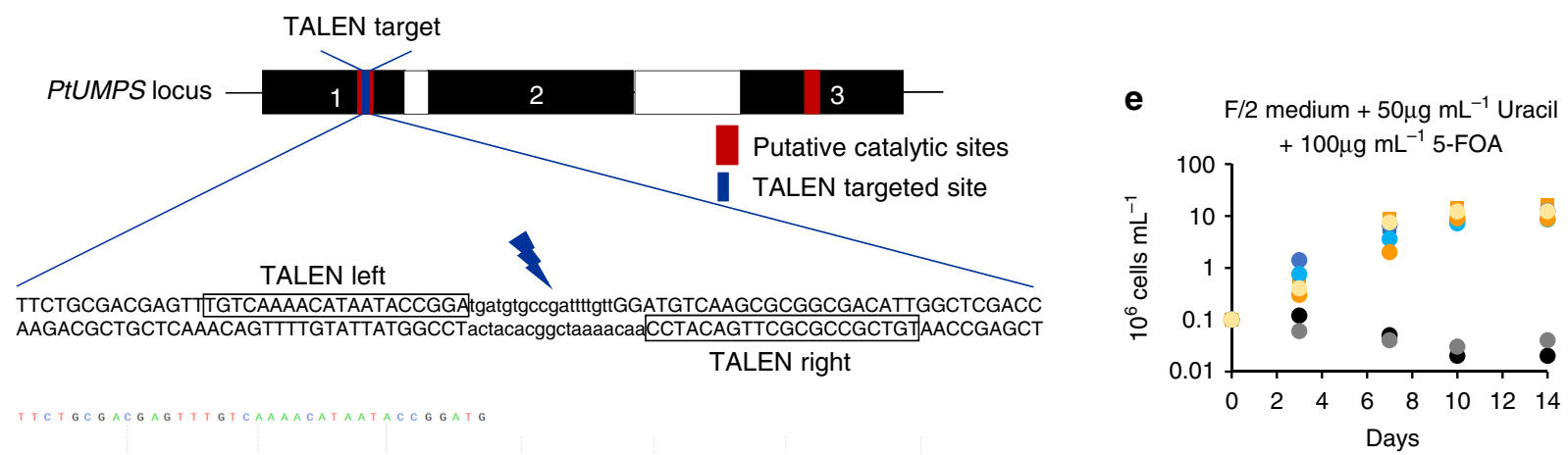

b

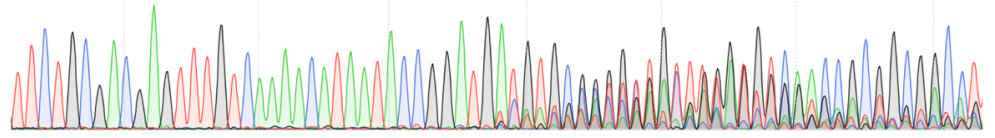

C
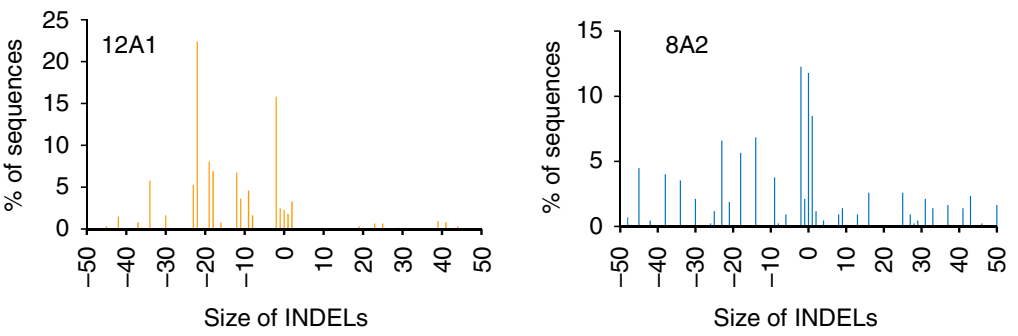

d

WT Allele1 GAGTTTGTCAAAACATAATACCGGAtgatgtgccgatttgttGGATGTCAAGCGCGGCGACATTGG

WT Allele2 GAGTTTGTCAAAACATAATACCGGAtgatgtgccgatttgttGGATGTCAAGCGCGGCGACATTGG

8A2 1 Allele1 GAGTTTGTCAAAACATAA---o---o--tGGATGTCAAGCGCGGCGACATTGG

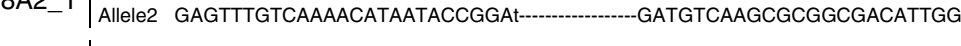

8A2_8 |Allele1 No amplification

12A1_2 $\begin{aligned} & \text { Allele1 No amplification } \\ & \text { Allele2 }\end{aligned}$ GAGTTTGTCAAAACATAATACCGGAtgatgtgcCcgatttgittGGATGTCAAGCGCGGCGACATTGG

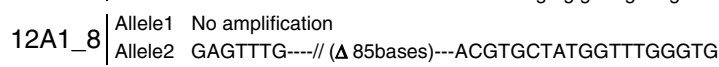

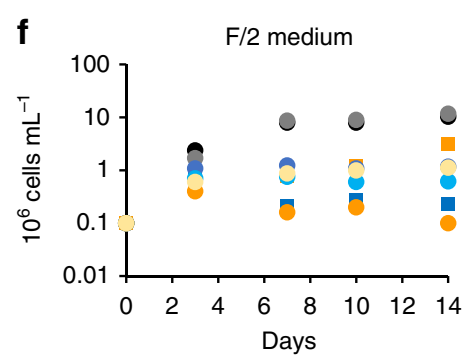

g $\quad \mathrm{F} / 2$ medium $+50 \mu \mathrm{g} \mathrm{mL}^{-1}$ Uracil

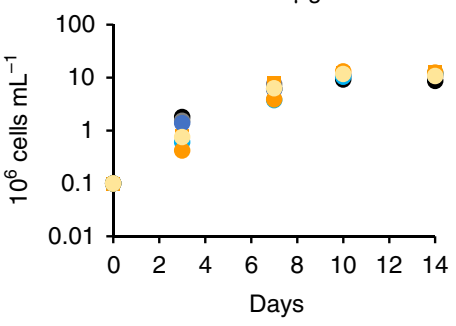

Fig. 1 Characterization of PtUMPS knock-out strains generated using a TALE nuclease. a Structure of the PtUMPS locus. The exons are represented as black boxes, with the putative catalytic sites in red and the TALEN-targeted site in blue. The half-sites recognized by each TALEN are boxed. b Sequence chromatogram of a PCR product amplified from strain 12A1, showing the presence of heterogeneous sequences surrounding the TALEN cutting site. c INDEL spectrum and frequency predicted by the Track INDELs by DEcomposition (TIDE) algorithm in the two 5-FOA-resistant colonies, 8A2 and 12A1. d Example of mutagenic events through the alignment of the wild type and 8A2_1, 8A2_8, 12A1_2, and 12A1_8 subclones DNA sequences at the PtUMPS locus. e-g Representative growth experiments from two independent repeats performed over 14 days with the parental NCMA strain (black circles), the $2 \mathrm{~A} 3$ control strain transformed with the NAT vector only (gray circles), two 5-FOA-resistant populations (8A2 dark blue squares and 12A1 dark orange squares), and four subclones: 8A2_1 (dark blue circles), 8A2_8 (light blue circles), 12A1_2 (dark orange circles) and 12A1_8 (yellow circles), e F/2 medium supplemented with $50 \mu \mathrm{g} \mathrm{mL} \mathrm{L}^{-1}$ uracil and $100 \mu \mathrm{g} \mathrm{mL}^{-1} 5$-FOA, f classical F/2 medium, or $\mathbf{g} \mathrm{F} / 2$ supplemented with $50 \mu \mathrm{g} \mathrm{mL}-1$ uracil. Y-axis e-g presents cell density, expressed as million cells per $\mathrm{mL}$, on a logarithmic scale

Antibiotic-free Cas9-driven inactivation of the PtUMPS gene. Other prerequisites for our proposed DNA-free genome editing strategy consist of demonstrating that (i) a PtUMPS KO can be achieved efficiently without introducing an exogenous antibiotic resistance cassette, solely relying on the $5-\mathrm{FOA}^{\mathrm{R}}$ phenotype for selection and (ii) multiple genes can be efficiently inactivated in a single step, including PtUMPS, which serves as an endogenous co-selection marker. The versatility of the CRISPR-Cas9 system, associated with its potential for multiplexing, prompted us to evaluate its capacity to achieve this strategy. We designed three gRNAs directed towards sequences situated within PtUMPS exons 1 and 3 (gUMPS1 and gUMPS4, Fig. 2a), which encode the putative catalytic sites, and exon 2 (gUMPS3) using the CRISPOR web-based tool ${ }^{25}$.

We tested whether the selected gRNAs can efficiently target the Cas9 protein towards the PtUMPS locus by first performing in vitro assays. gRNAs were complexed to the recombinant Cas 9 protein (Supplementary Fig. 3), thereby forming ribonucleoproteins (RNPs). The complexes were then mixed with a PtUMPS amplicon comprising the target sites. All RNP complexes efficiently catalyzed double strand DNA cleavage of the PtUMPS fragment, whereas Cas9 or gRNAs alone did not (Supplementary Fig. 3).

Three separate sgRNA expression vectors were assembled for gUMPS1, gUMPS3, and gUMPS4, as previously described ${ }^{10}$, to examine the in vivo functionality of these gRNAs. Individual sgRNA expression plasmids were co-bombarded into WT cells together with a plasmid encoding the Cas9 nuclease and another carrying the $\mathrm{NAT}^{\mathrm{R}}$ cassette. $\mathrm{NAT}^{\mathrm{R}}$ colonies appeared under all transformation conditions after 2-3 weeks. The presence of the Cas9-encoding gene was verified in all transformants by PCR before the PtUMPS locus was sequenced. From 29 to $100 \%$ of the screened colonies showed TM at the PtUMPS locus, depending on the gRNA used (Fig. 2b). Examples of mutagenic events are 
a

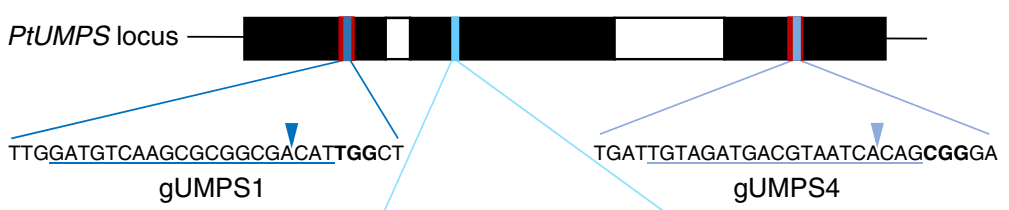

TGCCAAGCTTGTTGGCTCGGAATGGGCT gUMPS3

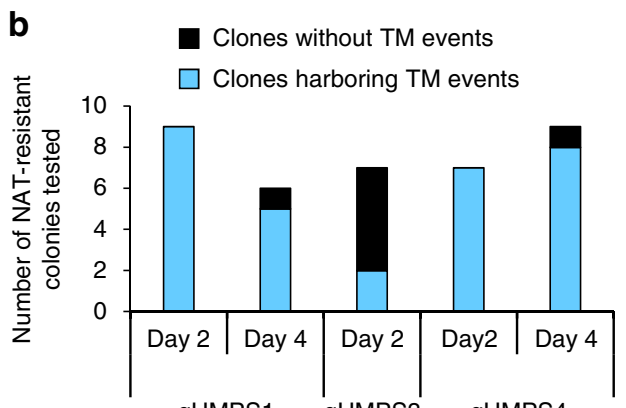

gUMPS1

gUMPS4

Fig. 2 CRISPR/Cas9-induced knock-out mutations at the PtUMPS locus. a Schematic representation of the position and sequence targeted by each gRNA: gUMPS1, gUMPS3, and gUMPS4. The 20-nt region of the target site is underlined and the PAM (Protospacer Adjacent Motif) is shown in bold letters. b Efficiency of targeted mutagenesis induced by the delivery of the NAT resistance cassette, gRNA, and Cas9 encoding vectors into WT Phaeodactylum cells after selection on NAT containing medium

illustrated in Supplementary Figs. 4, 5 and 6. We only detected one allele for most clones, again indicative of LOH; INDELs appeared close to the PAM sequences on the other allele, as expected. Thus, this step validated gUMPS1, gUMPS3, and gUMPS4 function in vivo.

Next we examined whether it was possible to obtain 5-FOAresistant colonies after direct selection of the cells transformed with plasmids encoding the Cas9 protein and either gUMPS1 or gUMPS4. Cells were transformed with either of these gRNA encoding plasmids and directly spread onto selective medium containing uracil and 5-FOA. Twelve $5-\mathrm{FOA}^{\mathrm{R}}$ clones appeared. Their genotypic characterization confirmed the presence of mutations adjacent to the PAM sequence in 10 out of 12 cases (Supplementary Table 1), demonstrating that direct selection on 5-FOA can lead to the identification of mutagenic events. The two remaining clones were not mutated at the PtUMPS loci, suggesting that they were false positives.

\section{Demonstration of RNP delivery in Phaeodactylumtricornutum.} We established the proof of concept of DNA-free genome editing in diatoms by adapting a proteolistic protocol established by Martin-Ortigosa and Wang ${ }^{26}$ to deliver Cas9-gRNA RNPs into Phaeodactylum cells (Fig. 3a). We first evaluated the frequency of targeted mutagenesis induced by the Cas9-gUMPS1 RNP complex in the absence of selection. To achieve that, P. tricornutum cells were bombarded with a dose response of $0,2,4$, and $8 \mu \mathrm{g}$ of Cas9-gUMPS1 complex. We did not expect a RNP transformation efficiency higher than $10^{-6}$ in the absence of selection, as in the case of DNA delivery it is necessary to co-transform 100 million cells with a plasmid encoding an antibiotic resistance to obtain 30 antibiotic-resistant clones ${ }^{6,9,10}$. To test this hypothesis, we collected the cells growing in the absence of selection at four days post-bombardment and quantified the mutagenesis at the PtUMPS locus using locus-specific PCR followed by deep sequencing (Supplementary Fig. 7). As positive controls, different amounts of a monoallelic mutant strain carrying a 1nt deletion at the gUMPS1 target site were mixed with WT cells to get cell-tocell ratios of $100 \%, 10 \%, 1 \%, 0.1 \%$ and $0 \%$. Whereas mutagenic events were detected in the positive controls at the expected frequencies, no induced mutagenic event was detected in the samples corresponding to cells bombarded with Cas9-gUMPS1, which reveals a TM frequency of less than $0.4 \%$. This result suggests that in the absence of selection, it is quasi impossible to produce DNA-free transgenic strains. We decided to evaluate our ability to generate such mutant strains using the PtUMPS counter-selectable marker.

The gUMPS1, gUMPS3, and gUMPS4 guides were individually mixed with the Cas9 protein to form the RNP complexes. Four micrograms of individual Cas9-gRNA RNP complex was coated onto gold particles and bombarded into WT Phaeodactylum cells using a helium gene gun. Cultures were plated onto 5-FOA plus uracil containing medium two or four days post-bombardment. After three to four weeks, 5-FOA resistant colonies appeared for all conditions, except when cells were bombarded with Cas9 only as a negative control (Supplementary Table 2). A second round of selection confirmed that four of four colonies obtained with the gUMPS4 RNP complex, one of three with the gUMPS1 RNP complex, and one of two with the gUMPS3 RNP complex could grow on 5-FOA uracil medium. These $5-\mathrm{FOA}^{\mathrm{R}}$ strains were then analyzed for TM at the PtUMPS locus (Supplemental Table 1). We failed to amplify the PtUMPS locus in the gUMPS1-derived and gUMPS3-derived strains, suggesting that a large deletion may have occurred. However, we observed TM close to the PAM in all gUMPS4-derived strains. This shows that transient exposure to the RNPs is sufficient to induce TM at an endogenous locus. Thus, we generated knock-out transgenic strain without introducing exogenous DNA into the cells.

Optimizing multiplex DNA-free genome editing. Our main objective was to inactivate multiple genes of interest in a single step, relying on the PtUMPS KO 5-FOA-resistance phenotype for positive selection. We started with the assumption that double KO strains could be generated by bombarding cells with a combination of RNP complexes, targeting the PtUMPS gene and another gene of interest (Fig. 3a). We selected the gene encoding Aureochrome 1a as an example (PtAureola, Phatr3_J8113); PtAureola is a photoreceptor that participates in blue light perception $^{22}$ and whose TALEN-mediated biallelic genetic KO was previously reported ${ }^{9,11}$. The gRNAs targeting PtAureola (gAureo1a2 and gAureola3) were selected following the same in silico criteria as for PtUMPS (Supplementary Table 3). Their activities were first verified in vitro using the RNP-based assay (Supplementary Fig. 8). Next, WT Phaeodactylum cells were simultaneously bombarded with multiple RNP complexes, targeting both PtUMPS and PtAureola (Fig. 3a).We used two gRNAs per target (gUMPS1 and gUMPS3, targeting the PtUMPS locus; gAureola2 and gAureola3, targeting the PtAureola locus) and mixed them independently with recombinant Cas9 protein to increase our chances of obtaining TM at both loci. The four RNP complexes were then combined (Fig. 3a). Cells were then co-bombarded with $8 \mu \mathrm{g}$ of RNP complexes (corresponding to $2 \mu \mathrm{g}$ of each RNP) and transferred onto 5-FOA uracil medium two days postbombardment. Clones were first analyzed for TM at the PtUMPS locus (Fig. 3b). Among the $195-$ FOA $^{\mathrm{R}}$ colonies that appeared, 17 (89\%) showed TM at the PtUMPS locus: 4/17 (23\%) with mutagenic events on both alleles and 13/17 (76\%) with mutagenic 
a
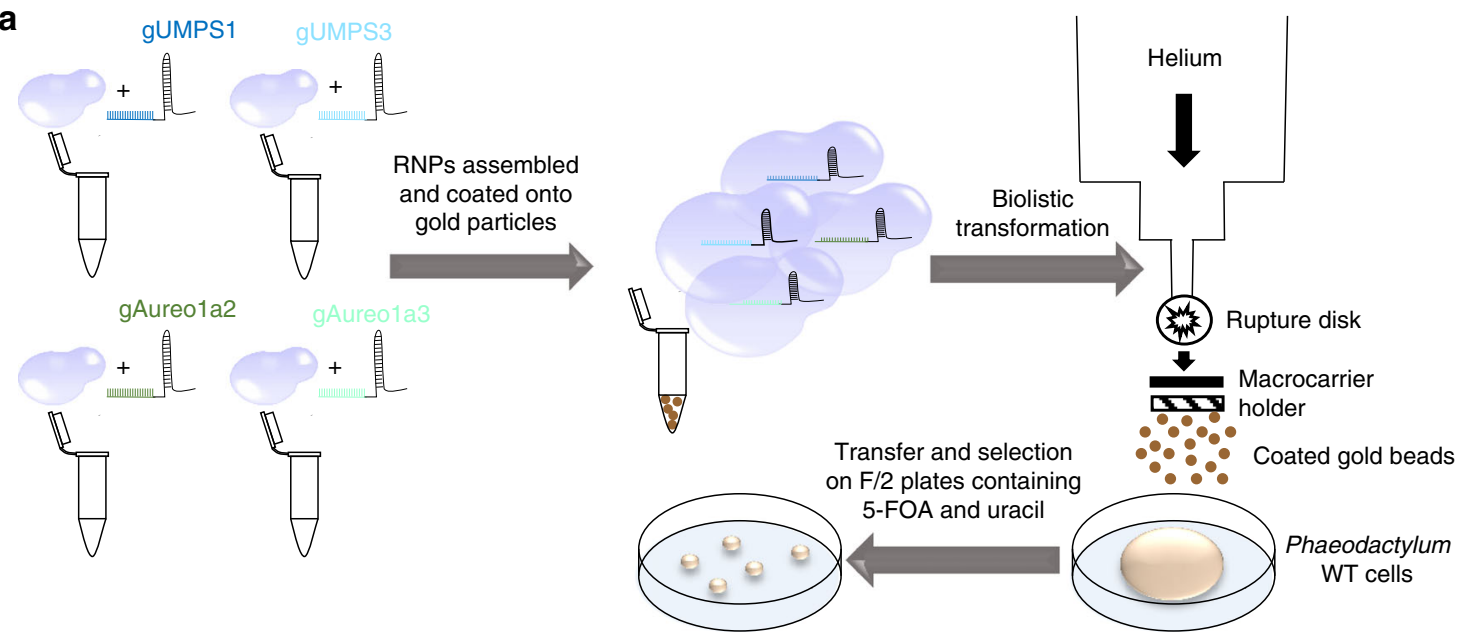

b
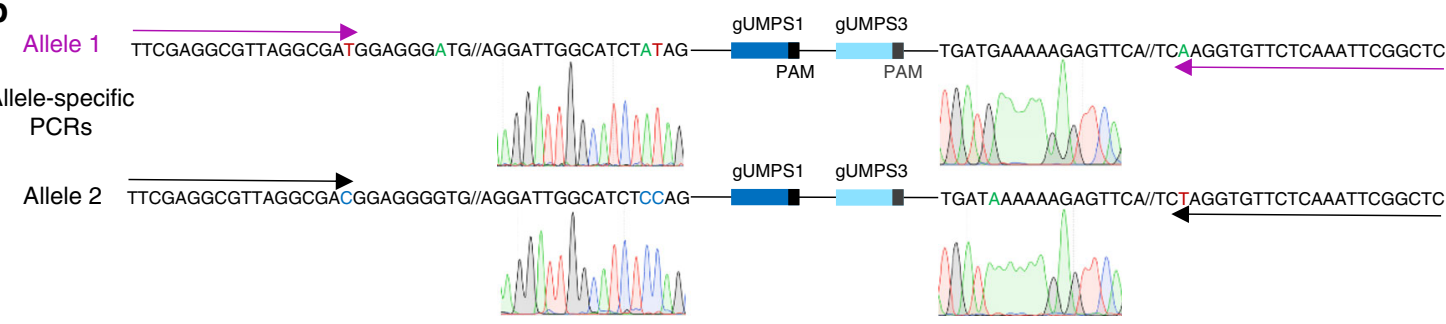

C

Mutations at the PtUMPS target sites

d
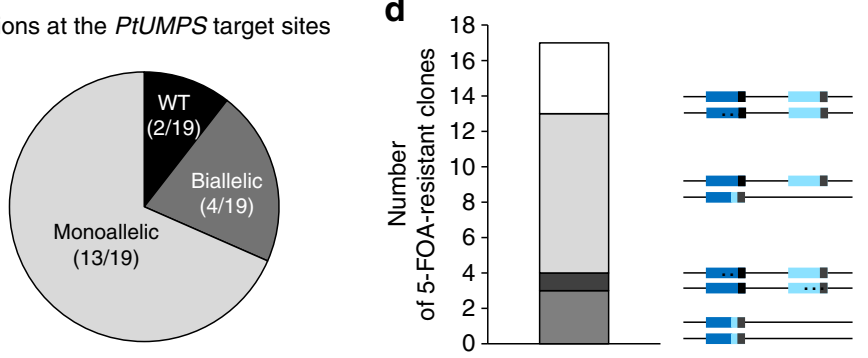

Small INDELs in one allele

Large deletion in one allele

Small INDELs in both alleles

Large deletion in both alleles

e Mutations at the PtAureo1a target sites
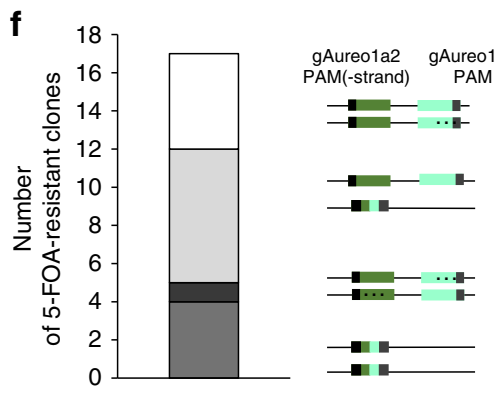

Small INDELs in one allele

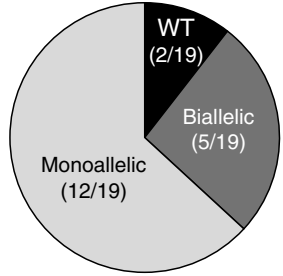

Fig. 3 Multiple gene knock-outs using a DNA-free RNP genome editing approach. a Outline of the genome-editing workflow used here. The first step consists of generating four independent RNP complexes, two of them targeting PtUMPS and the two others targeting PtAureo1a or any other gene of interest. Each RNP complex is prepared in a separate tube by incubating the Cas9 protein with pre-annealed crRNA::tracrRNA complexes (crRNA: base pairing site, tracrRNA: trans-activating crRNA). Next, the four RNP complexes are combined and loaded onto gold particles. The third step consists of the delivery of these coated gold beads into Phaeodactylum cells using a biolistic apparatus. Finally, after two to four days, the transformed cells are re-plated onto selective medium, here F/2 medium supplemented with uracil and 5-FOA. After three to four weeks, 5-FOA-resistants colonies appear and are analyzed for the presence of mutagenic events. $\mathbf{b}$ Each allele of the PtUMPS gene was amplified by allele-specific PCRs from the obtained 5-FOA resistant colonies and sequenced. c Frequency of colonies harboring no mutagenic event at the PtUMPS loci (black sector on the pie chart) or a mutagenic event on one allele (light gray sector) or both alleles (dark gray sector). d Additional information on the nature of the mutagenic events is reported, with four classes of mutagenic events being illustrated: small INDELs in one allele, the second being WT (white bar); large deletion corresponding to the DNA sequence between the two targeted sites in one allele, the second allele being WT (light gray bar); small INDELs in both alleles (dark gray bar); deletion of the DNA fragment between the two targeted sites in both alleles (medium gray bar). e Frequency of targeted mutagenesis of the PtAureola target sites (black sector: no TM detected, light gray sector: monoallelic mutants, dark gray sector: biallelic mutants). All clones mutated in PtUMPS also carried a mutation in at least one of the PtAureola alleles. $\mathbf{f}$ Distribution for the various classes of mutagenic events at the PtAureola loci 
events on one allele only, the second carrying the WT sequence (Fig. 3c). Amplification of PtUMPS resulted in a clearly shorter PCR product for $11 / 17$ (65\%) of the clones (Fig. 3d, Supplementary Fig. 9). Sequencing of these PCR products revealed deletion of the fragment between gUMPS1 and gUMPS3, indicating that DSBs were created at both target sites (Fig. 3d, Supplementary Fig. 9). The PtAureola locus of the 195 -FOA ${ }^{\mathrm{R}}$ colonies was amplified by PCR and sequenced. The two strains that did not show TM of PtUMPS also showed no TM of PtAureo1a. Further analysis revealed that $100 \%$ of the PtUMPSmutated clones also displayed a mutagenic event at the PtAureola locus; 5 of 17 carried mutations on both alleles (Fig. 3d, e). Thus we achieved simultaneous multiple gene knock-outs in diatoms.

Extrapolation to another selectable marker. We evaluated the transferability of this approach by investigating another putative selectable marker, the Adenine Phosphoribosyl Transferase (APT) gene, for which inactivation confers 2-FA resistance in several organisms, including plants ${ }^{21,27}$. APT normally contributes to adenine recycling as part of the nucleotide salvage pathway ${ }^{27}$. We identified a single potential APT encoding gene in the Phaeodactylum genome (Phatr3_J6834, hereafter referred to as PtAPT; UniProt B7G7K1), based on sequence homology of its translation product with the Physcomitrella patens APT protein (PpAPT, UniProt: Q45RT2) ${ }^{21}$. Overall, PtAPT shares $41.7 \%$ amino-acid identity with PpAPT and 28.6\% with the Arabidopsis thaliana APT1 protein (AtAPT1, UniProt: P31166) (Fig. 4a). The phosphoribosyl transferase (PRT) type I domain, spanning amino-acid positions 28 to 132 in PtAPT, is well conserved.

Before proceeding further, we determined whether 2-FA was toxic to Phaeodactylum cells and at what concentrations. We observed a dose-response effect of 2-FA on growth, with complete inhibition at $10 \mu \mathrm{M}$ (Fig. 4b).

We designed gRNAs directed against PtAPT (gAPT1 and gAPT3) to target regions within exons 1 and 2, respectively (Fig. 4c). Both the gAPT1 RNP and gAPT3 RNP complexes efficiently catalyzed DSB DNA cleavage of a PtAPT amplicon in vitro (Supplementary Fig. 10). Either the gAPT1 or the gAPT3 RNP complex was then introduced into WT Phaeodactylum cells by proteolistic transformation. Cultures were plated onto selective medium containing $10 \mu \mathrm{M} 2$-FA and $5 \mathrm{mg} \cdot \mathrm{L}^{-1}$ adenine, two days post-bombardment (Fig. 4d). Eleven and five 2-FA ${ }^{\mathrm{R}}$ colonies appeared after two weeks for the gAPT1 and gAPT3 RNPs, respectively, whereas no colonies appeared in the negative control bombarded with the Cas9 protein alone. Among the $162-\mathrm{FA}^{\mathrm{R}}$ colonies, 15 (93\%) showed TM at the PtAPT locus (Fig. 4d), all of which carried mutations on both alleles, suggesting that biallelic $\mathrm{KO}$ is required to trigger resistance to 2-FA. Examples of mutagenic events are shown in the Supplementary Figs. 11 and 12. We thus demonstrated that inactivation of PtAPT confers 2$\mathrm{FA}^{\mathrm{R}}$, allowing the use of PtAPT as an endogenous positive selectable marker in Phaeodactylum.

We next evaluated the impact of knocking out PtAPT on growth in F/2 medium, F/2 medium supplemented with adenine, and F/2 medium supplemented with both 2-FA and adenine (Fig. 4e). As expected, WT cells were unable to grow in the presence of $10 \mu \mathrm{M} 2$-FA. The two tested APT KO strains were unaffected by the addition of 2-FA and grew normally in F/2 or F/ 2 plus adenine. Inactivating PtAPT did not negatively affect culture fitness, an important requirement for downstream applications.

We verified that the PtAPT KO can be used as a co-selection marker for multiplexing experiments by simultaneously bombarding WT Phaeodactylum cells with RNP complexes targeting the PtAPT and PtAureola loci (Fig. 4f). Two gRNAs were used per target (gAPT1 and gAPT3 targeting the PtAPT locus;
gAureola2 and gAureola3 targeting the PtAureo1a locus). Thus, cells were bombarded with $8 \mu \mathrm{g}$ of an RNP mixture consisting of all four complexes and spread onto selective medium containing 2-FA and adenine two days post-bombardment. Thirty-four 2$\mathrm{FA}^{\mathrm{R}}$ clones appeared within two weeks. All showed TM at the PtAPT locus. Amplification of PtAPT resulted in shortened PCR fragments for $47 \%$ of the colonies (16/34), indicating that the deletion occurred between the gAPT1 and gAPT3 target sites (Supplementary Fig. 13). These results were confirmed by sequencing 29 of these 34 clones. We also investigated the presence of mutagenic events at the PtAureola locus. Sixty-five percent (19/29) of the colonies harboring PtAPT mutations also showed mutagenic events at the PtAureola locus and 52\% (10/19) of them exhibited a deletion of the fragment between the two target sites. Interestingly, 52\% (10/19) of the PtAureo1a mutants were mutated on both alleles, leading to the generation of PtAureola KO strains (Fig. 4f).

We next determined whether three genes can be simultaneously knocked-out. Cells were bombarded with a mixture of six RNP complexes, two RNPs targeting PtAPT, two PtUMPS, and two PtAureo1a, and selected on 2-FA medium supplemented with adenine and uracil. Fourteen 2-FA-resistant colonies appeared. Thirteen of the fourteen colonies (93\%) showed a mutagenic event at the PtAPT locus (Fig. 5). Among the 13 clones, five were mutated at both PtAPT and PtAureola, two at both PtAPT and PtUMPS, and two at all the three loci. The genotype of these clones is summarized in Fig. 5.

We should point out that the selection of 2-FA resistant clones was achieved in all of our independent trials $(n=7)$ whereas the selection of 5-FOA-resistant clones failed in three experiments out of eight. Such a disparity has already been reported in other organisms where $\mathrm{pH}$, light, and temperature have been shown to influence 5-FOA selection strength. The major point is that we have succeeded in generating multiple PtAureo1a knock-out strains in the three independent multiplexed experiments described in Supplementary Table 4. Our results show that this genome-editing approach is a powerful tool to simultaneously inactivate multiple genes without the use of DNA and antibiotics.

To go one step further, we phenotypically characterized eleven RNP-derived transformants from several independent experiments that were mutated in PtUMPS and/or PtAPT and/or PtAureo1a (Supplementary Fig. 14a). First we tested their resistance to 5-FOA, as well as 2-FA (Supplementary Fig. 14b). For all these strains, sensitivity or resistance to different media reflected genotypes well. Next, we quantified the amount of PtAureola in the strains by Western Blotting (Supplementary Fig. 15). Whereas we did not detect any PtAureola protein in the samples derived from bi-allelic PtAureo1a mutant strains, we clearly detected it in protein samples derived from the tested monoallelic PtAureola strain and the positive controls. We additionally performed growth experiments and observed that the cells mutated in PtAureola had lower (20\% maximum) growth rates compared to the parental strain or the PtUMPS and PtAPT single mutants (Supplementary Fig. 16). Finally, we evaluated the photo-physiological impact of the PtAureola mutation. This gene encodes for a blue-light photoreceptor of which knock-out strongly affects cells ability to dissipate excess light energy as heat upon a shift from low to high irradiances ${ }^{9}$. This can be quantified by measuring the Non-Photochemical Quenching (NPQ) of chlorophyll fluorescence? ${ }^{9}$. As previously reported, biallelic $P$. tricornutum PtAureola mutant strains generated by TALE nucleases display reduced NPQ capacities compared to wildtype $^{9}$. We performed similar NPQ capacity measurements. Whereas no NPQ phenotype was observed in the monoallelic PtAureola mutant strain tested here and in the PtAPT and PtUMPS knock-out strains, we measured a 50-73\% reduction of 
a

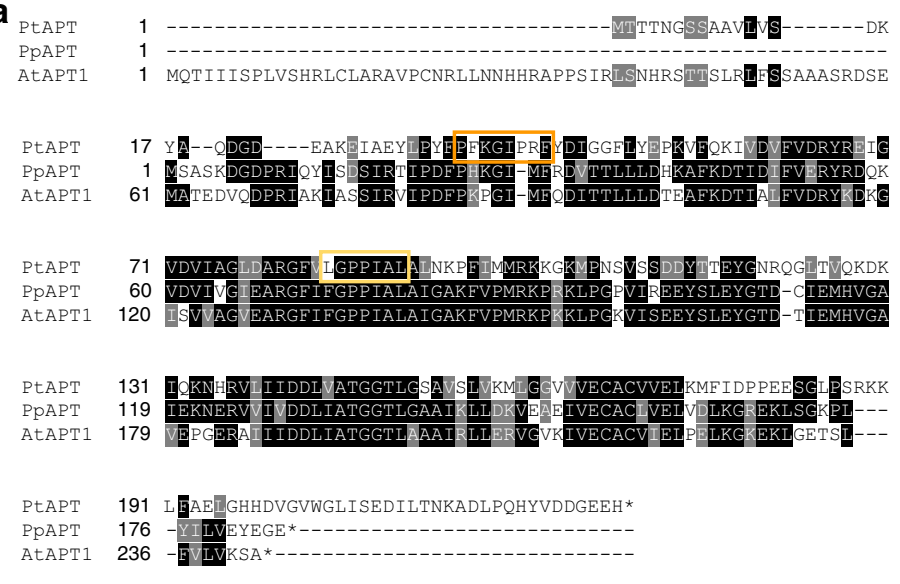

C

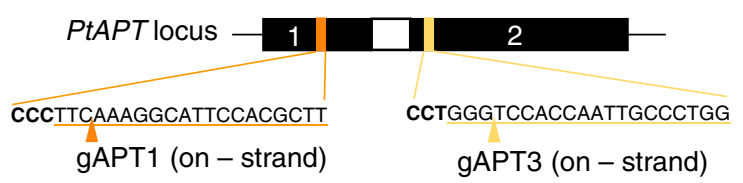

b

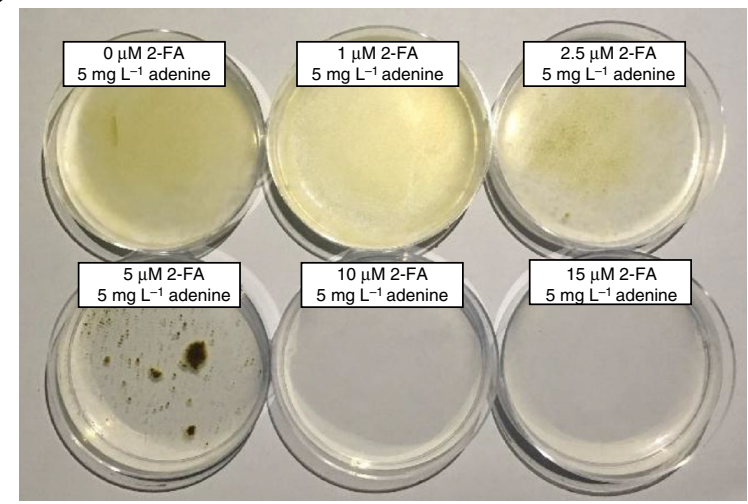

d

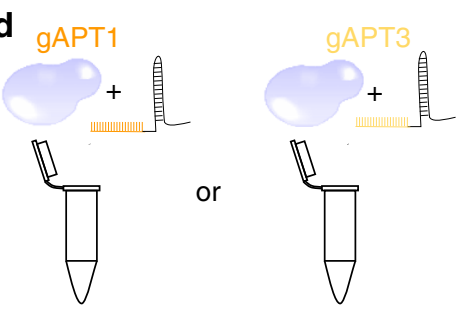

e

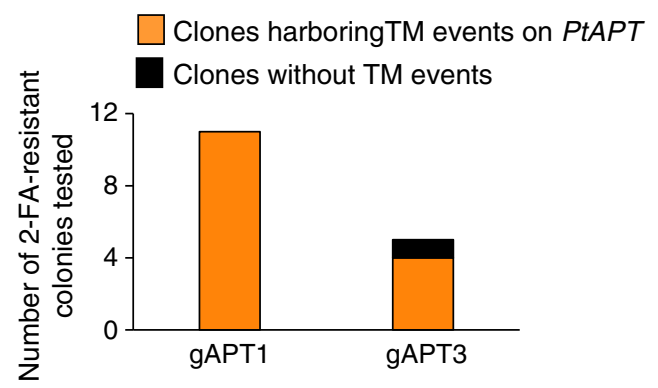

g Multiplexing experiment: mutations at the PtAureo1a loci

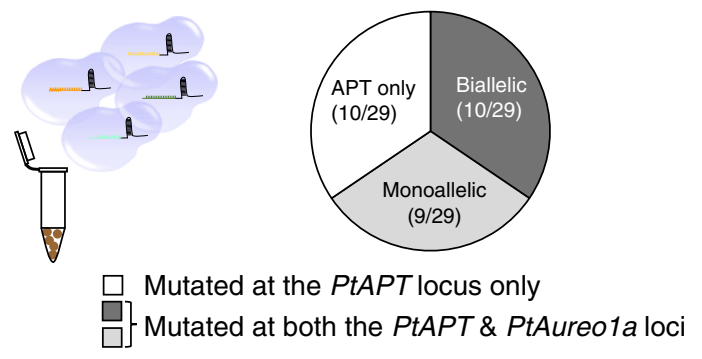

f

$\mathrm{F} / 2$ medium $+5 \mathrm{mg} \mathrm{L}^{-1}$ adenine $+10 \mu \mathrm{M}$ 2-FA

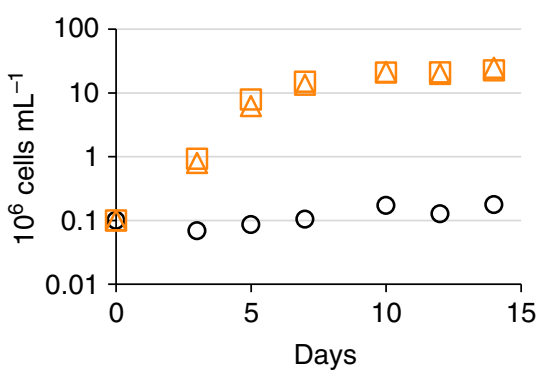

O NCMA

$\square$ APT KO clone 1

$\triangle$ APT KO clone 2

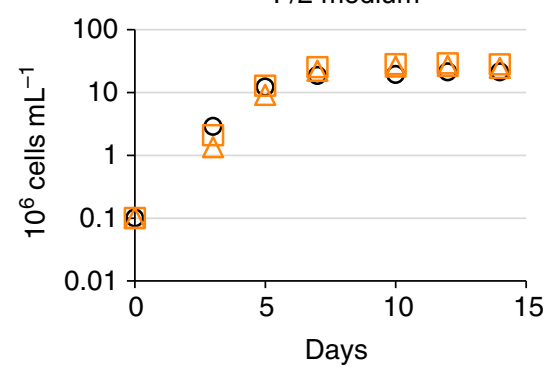

O NCMA

$\square$ APT KO clone 1

$\triangle$ APT KO clone 2

Fig. 4 PtAPT, another endogenous selection marker for DNA-free genome editing. a Alignment of the APT protein sequences from Arabidopsis thaliana (AtAPT1), Physchomitrella patens (PPAPT), and P. tricornutum (PtAPT). Conserved amino acids are highlighted. Orange boxes represent the gRNA targeting sites. b Sensitivity of NCMA (WT) P. tricornutum cells to chronic exposure to various concentrations of 2-FA in the presence of adenine. c Structure of the PtAPT locus. Exons are represented as black boxes, introns as white boxes. Sequences targeted by gAPT1 (dark orange) and gAPT3 (light orange), both of them on the reverse complementary (-) strand, are indicated with their Protospacer Adjacent Motives shown in bold font. d RNPs were prepared by complexing either of the corresponding gRNAs to recombinant Cas9. e In vivo evaluation of targeted mutagenesis induced by the Cas9-gAPT1 or Cas9-gAPT3 RNP complexes. Phaeodactylum cells bombarded with the corresponding RNP were selected on F/2 medium containing $10 \mu \mathrm{M} 2-\mathrm{FA}$ plus $5 \mathrm{mg} \mathrm{L}^{-1}$ adenine. The PtAPT locus of the generated 2-FA-resistant colonies was amplified by PCR and sequenced. $\mathbf{f}$ Representative growth experiment from two independent repeats performed with two bi-allelic APT mutants and the NCMA parental cell grown in F/2 plus 2-FA and F/2 alone. $\mathbf{g}$ Frequency of targeted mutagenesis of the PtAureola loci in colonies that were simultaneously bombarded with two RNPs against PtAPT (gAPT1 and gAPT3) and two RNPs against PtAureo1a (gAureo1a2 and gAureo1a3) 

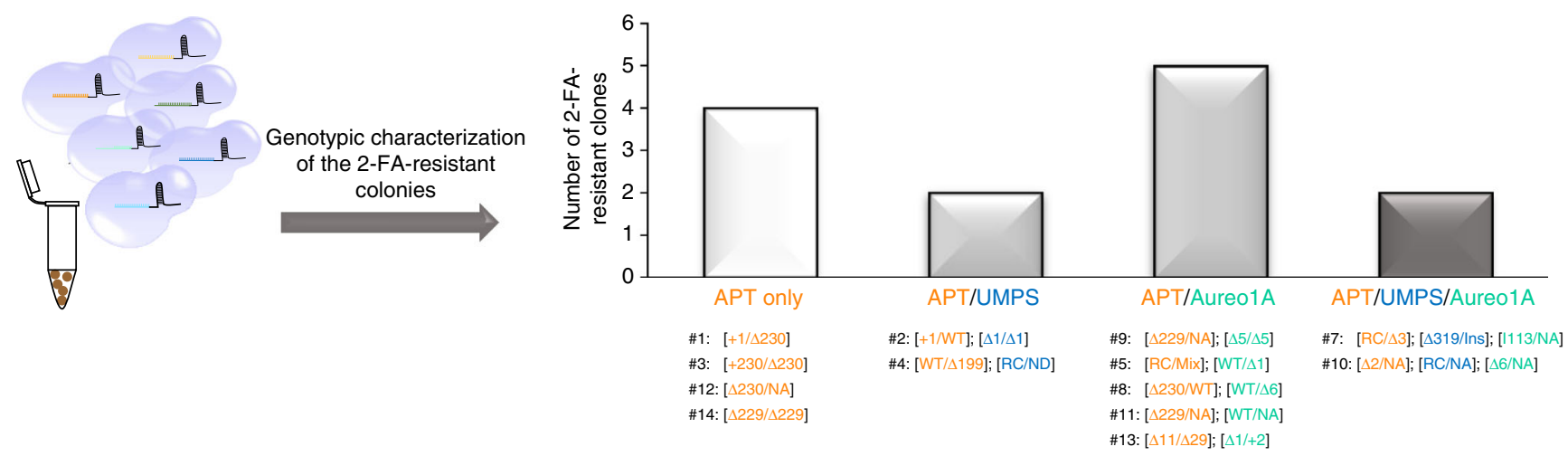

Fig. 5 One-step generation of triple knock-out strains. WT Phaeodactylum cells were simultaneously bombarded with six RNP complexes, each directed against one specific target at either the PtAPT, PtUMPS, or PtAureo1a loci. After two days, cultures were transferred onto 2-FA selective medium supplemented with adenine and uracil. Fourteen 2-FA resistant clones (referred to as strains AUA\#1 to \#14) were obtained and screened for the presence of mutagenic events at the six target sites. Bar plots present the number of 2-FA resistant colonies harboring mutations at the PtAPT loci only (white bar), at both the PtAPT and PtUMPS loci or the PtAPT and PtAureola loci (light gray bars), or at all three loci (dark gray bar). The genotypes of the corresponding clones are presented underneath the graph and written in orange font for PtAPT, blue for PtUMPS and green for PtAureo1a. RC reverse complement insertion. NA not amplifiable, Mix mosaic, ND not determined

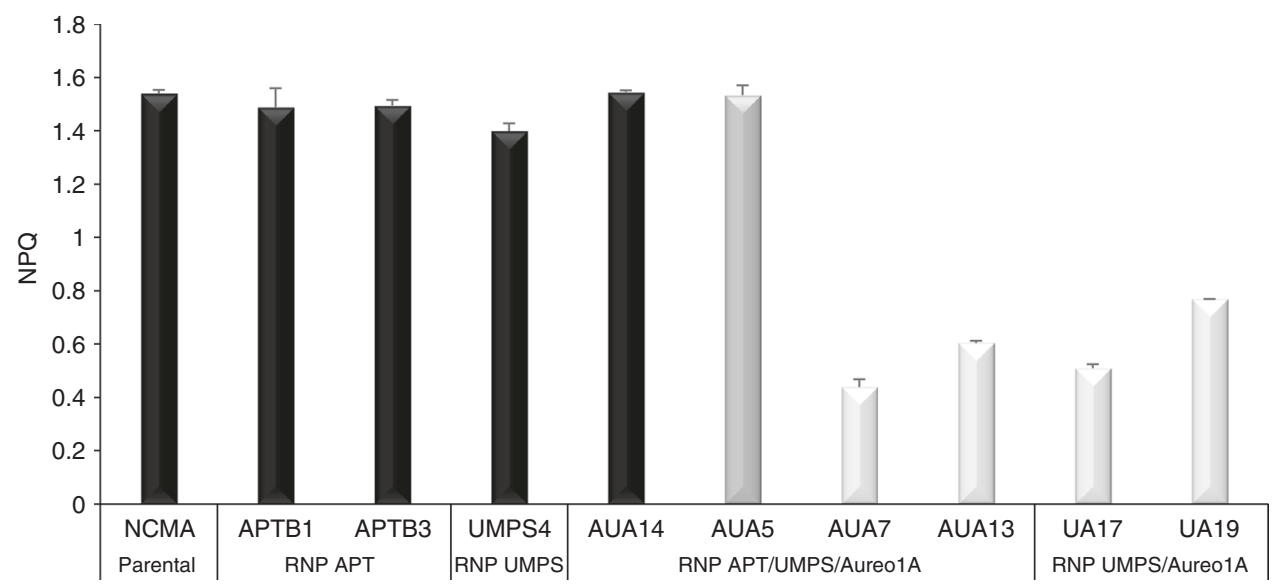

Fig. 6 Non-photochemical quenching in RNP-derived PtAureola mutant strains. The non-photochemical quenching (NPQ) of chlorophyll fluorescence was measured after ten minutes of illumination with $700 \mu \mathrm{mol}$ photons $\mathrm{m}^{-2} \mathrm{~s}^{-1}$ of actinic red light. The parental NCMA strain, two PtAPT single mutants (APTB1 and APTB3), a PtUMPS single mutant (UMPS4) and strain AUA14 showing no TM at the PtAureola loci served as controls (black bars). Several strains derived from multiplexing experiments-including a PtAureola monoallelic mutant (AUA5; dark gray bar), as well as PtAureo1a biallelic mutants (AUA7, AUA13, UA17, and UA19; light gray bars)-were characterized. The genotypes of these strains are shown in Supplementary Fig. 14. Data are the average of $n=2$ independent repeats, bars show SD

NPQ in all RNP-derived biallelic PtAureo1a mutants tested (Fig. 6). Therefore, we confirmed the loss of function of the generated PtAureola knock-out strains, which reveals the power of our approach to study gene function.

\section{Discussion}

Here, we established a DNA-free approach that solves the important issues of random DNA integration into the diatom genome and long-term nuclease expression. This was achieved by combining RNPs targeting one endogenous marker gene with RNPs targeting another gene of interest, successfully generating dozens of transgenic strains free of foreign DNA, without the use of antibiotics. Moreover, the same strategy allowed the creation of triple-gene knock-out strains in a one-step procedure.

This is a valuable research tool for algae genome engineering that should open the way to the study of putative redundant functions of multiple gene family members. For example, it could be suitable to investigate the role of specific light-harvesting complexes of the LHCX family during exposure to excess light $^{28}$ or the contribution of single aureochromes to photoacclimation $^{11}$.

Our approach also represents a technical breakthrough for industrial biotechnology, as it will permit the simultaneous $\mathrm{KO}$ of multiple endogenous pathways to redirect metabolism towards the production of biomolecules of interest. Moreover, the success of our strategy was associated with the identification of the PtUMPS and PtAPT genes and the validation that their inactivation confers resistance to specific molecules. The use of such markers represents an attractive alternative to conventional antibiotic-resistance genes and solves the environmental issue posed by the dissemination of such resistance genes into other organisms through horizontal transfer.

The methodology we have developed addresses the global effort to exploit non-integrative expression of the CRISPR-Cas9 system. A recent report based on episome delivery provided a significant technological advance[12]; however, this system may not be sufficient to solve the challenge of limiting the amount and duration of nuclease exposure. Indeed, cells are exposed to 
nucleases for several weeks, the necessary time for the appearance of antibiotic-resistant colonies. To date, no studies have investigated a potential deleterious impact of long-term Cas9 expression in microalgae. If that were the case, the transient expression of the CRISPR-Cas9 system described here could decrease undesired mutations compared to long-term expression ${ }^{29}$. A major benefit of RNP delivery is its simplicity and ease of implementation, as it eliminates all subcloning steps, saving considerable time relative to traditional genome-editing approaches.

Another attractive perspective relies on the fact that the UMPS and APT markers are well conserved within the microalgae phylogenetic tree and among other eukaryotic groups, making it highly likely that the method is extendable to other organisms. This is particularly important for hard-to-transfect species or those for which no biobricks (promoters, terminators) are available.

\section{Methods}

Culture conditions. The Phaeodactylum tricornutum strain CCMP2561 (NCMA) was grown axenically at $20^{\circ} \mathrm{C}$ in vented cap flasks containing silica-free $\mathrm{F} / 2$ medium (Sigma G0154) with 40\% Sea Salts (Sigma S9883). Incubators were equipped with white neon light tubes providing an illumination of approximately $120 \mu \mathrm{mol}$ photons $\mathrm{m}^{-2} \mathrm{~s}^{-1}$ and a photoperiod of $12 \mathrm{~h}$ light $/ 12 \mathrm{~h}$ dark.

Selection procedures. For the Nourseothricin (NAT) selection procedure, cells transformed with the NAT selection plasmid were collected two or four days posttransformation and spread on two F/2 agar plates with $300 \mu \mathrm{g} \mathrm{ml}^{-1}$ NAT (Werner Bioagents). After three weeks, colonies were re-streaked on fresh $10-\mathrm{cm} 1 \%$ agar plates containing $300 \mu \mathrm{g} \mathrm{ml}^{-1} \mathrm{NAT}$. For selection on 5-FOA, NAT-transformants were then transferred onto $\mathrm{F} / 2$ agar plates containing $50 \mu \mathrm{g} \mathrm{mL} L^{-1}$ uracil and 100 $\mu \mathrm{gL}^{-1} 5$-FOA. In RNP experiments, cells were spread two or four days posttransformation onto $\mathrm{F} / 2$ agar media supplemented for selection with $50 \mu \mathrm{g} \mathrm{mL} \mathrm{L}^{-1}$ uracil and $100 \mu \mathrm{g} \mathrm{mL}^{-1} 5$-FOA, to detect UMPS-mutated colonies, or onto $\mathrm{F} / 2$ agar media supplemented with $5 \mathrm{mg} \mathrm{L}^{-1}$ adenine and $10 \mu \mathrm{M}$ of 2-FA, to detect APTmutated colonies. In the RNP experiments simultaneously targeting the UMPS, APT, and Aureochrome1A genes, cells were selected on F/2 medium supplemented with $10 \mu \mathrm{M} 2$-FA, $5 \mathrm{mg} \mathrm{L}^{-1}$ adenine, and $50 \mu \mathrm{g} \mathrm{mL}^{-1}$ uracil.

TALEN nuclease. The TALEN pairs were designed using TALE-NT (www.tale-nt. cac.cornell.edu ${ }^{30,31}$. We included two 20 -bp recognition sites separated by an 18 bp spacer (TGTCAAAACATAATACCGGAtgatgtgccgattttgttGGATGTCAAGCG CGGCGACA) ${ }^{30}$. All TALEN constructs were assembled through Golden Gate cloning and cloned into TALEN scaffolds ${ }^{6}$.

CRISPR-Cas9 design and constructs. Guide RNAs (gRNAs) were selected using the CRISPOR tool (http://crispor.tefor.net/) $)^{25}$, based on the Moreno-Mateos score and the absence of predicable off targets. Corresponding crRNA sequences are listed in Supplementary Table 3. The gRNAs were cloned under control of the U6 promoter in a vector derived from $\mathrm{pKSdiaCas} 9^{10}$. This vector was co-delivered with the Cas9 encoding plasmid pKSdiaCas9.

Biolistic transformation of TALEN or Cas9 and gRNA vectors. Cells $\left(1.5 \times 10^{8}\right.$ total) were collected from exponentially growing cultures and spread onto $1 \%$ agar plates containing F/2 medium with $20 \mathrm{~g} \mathrm{~L}^{-1}$ sea salt. Transformations were carried out $24 \mathrm{~h}$ later using the microparticle bombardment method adapted from Apt et al. ${ }^{32}$, with minor modifications. Gold particles $(0.6 \mu \mathrm{m}$ diameter, BioRad) were coated with DNA using $1.25 \mathrm{M} \mathrm{CaCl}_{2}$ and $20 \mathrm{mM}$ spermidine. As a negative control, beads were coated with $3 \mu \mathrm{g}$ NAT selection plasmid and $3 \mu \mathrm{g}$ empty vector. For the CRISPR-Cas9 experiment, the DNA mixture contained $3 \mu \mathrm{g}$ Cas9 expression vector (pKSdiaCas9) ${ }^{10}, 3 \mu \mathrm{g}$ U6-gRNA encoding plasmid, and $3 \mu \mathrm{g}$ NAT selection plasmid. As a negative control, beads were coated with a DNA mixture consisting of $3 \mu \mathrm{g}$ NAT selection plasmid, $3 \mu \mathrm{g}$ Cas 9 expression vector, and $3 \mu \mathrm{g}$ empty vector. A burst pressure of $1550 \mathrm{psi}$ and a vacuum of $25 \mathrm{Hg}$ were used.

Preparation of the crRNA-tracrRNA duplex. Purified crRNAs and tracrRNA were purchased from IDT. Duplexes were prepared following the manufacturer's instructions.

In vitro CRISPR-Cas9 RNP cleavage assay. DNA fragments containing the target site were amplified, purified, and eluted with RNase-free water. CRISPR-Cas9 RNP complexes were assembled using crRNA::tracrRNA duplexes and HiFi Cas9 (IDT \#1074181). Each RNP complex was combined with the corresponding DNA amplicon and the in vitro cleavage reaction allowed to proceed, following the manufacturer's instructions. Sequential RNase A and proteinase K treatments were performed before gel separation and visualization.
Biolistic bombardment of Cas9 RNP complexes. RNP complexes were assembled following the instructions of IDT and delivered to WT Phaeodactylum cells by particle bombardment. Briefly, $24 \mathrm{~h}$ before the experiment, cells $\left(1.5 \times 10^{8}\right)$ were spread onto $1 \%$ agar $\mathrm{F} / 2$ plates with $20 \mathrm{~g} \mathrm{~L}^{-1}$ sea salt and $50 \mu \mathrm{g} \mathrm{mL}^{-1}$ uracil, if targeting PtUMPS. For each shot, the equivalent of 4 or $8 \mu \mathrm{g}$ Cas 9 protein (for multiple targets, the total amount was split equally between the different RNPs) in a total volume of $8 \mu \mathrm{l}$ Cas 9 reaction buffer (NEB, B0386A) was mixed with $10 \mu \mathrm{l}$ gold nanoparticles ( $3 \mathrm{mg}, 0.6 \mu \mathrm{m}$ in diameter, Bio-Rad) washed twice with Cas9 reaction buffer. The coated particles were distributed slowly in a circle directly onto the macrocarrier, which was allowed to dry for $2 \mathrm{~h}$ before biolistic bombardment. A burst pressure of $1550 \mathrm{psi}$ and a vacuum of $25 \mathrm{Hg}$ were used.

Genotypic characterization. Cell lysates served as the PCR templates ${ }^{6}$. Primers listed in Supplementary Table 5 were used to amplify the various loci of interest. The Q5 High Fidelity DNA polymerase (New England BioLabs, USA) was used for general screening purposes. The highly selective HiDi DNA polymerase (myPOLS, Germany) was used for allele-specific PCR, together with primers ending with single nucleotide polymorphisms.

Deep sequencing. To quantify the frequency of mutagenic events, locus-specific PCR products were sequenced using NGS technology (S5 Thermofisher). In all conditions, 13,000-72,000 reads per sample were analyzed. The sequencing and the processing of NGS data was done by the GeT-Biopuces platform (INSA Toulouse) using the Ion Torrent Suite software analysis. The percentage of mutagenic event was calculated at position 145 ( 3 nt upstream of the gUMPS1 PAM) and at $+1-5$ bases surrounding this position. The frequency of total INDELs (including 1 base INDEL) was calculated, with a background noise level lower than $0.4 \%$. The frequency of INDELs larger than 1 base was also analyzed and in this condition the background noise level was extremely low $<0.06 \%$.

Cell growth experiments. For the growth experiments reported in Fig. 1e-g, cells were cultivated in $\mathrm{F} / 2$ medium for five days and then diluted to $1.10^{5}$ cells $\mathrm{mL}^{-1}$ in $\mathrm{F} / 2$ medium, $\mathrm{F} / 2$ medium supplemented with $50 \mu \mathrm{g} \mathrm{mL}^{-1}$ uracil, or $\mathrm{F} / 2$ medium supplemented with $50 \mu \mathrm{g} \mathrm{mL}^{-1}$ uracil and $100 \mu \mathrm{g} \mathrm{mL}^{-1}$ 5-FOA. For the growth experiment reported in Fig. 4, a similar procedure was followed, except that cells were diluted into $\mathrm{F} / 2$ medium, $\mathrm{F} / 2$ medium with $5 \mathrm{mg} \mathrm{L}^{-1}$ adenine, or F/2 medium with $5 \mathrm{mg} \mathrm{L}^{-1}$ adenine and $10 \mu \mathrm{M} 2$-FA after five days.

Measurement of non-photochemical quenching (NPQ). Cell suspensions in mid-exponential phase were adjusted to a chlorophyll a content of $1 \mu \mathrm{g} \mathrm{mL}^{-1}$ and NPQ was measured with an AquaPen-C AP 100 (Photon Systems Instruments, Brno, Czech Republic) using light pulses with an intensity of $2100 \mu \mathrm{mol}$ photons $\mathrm{m}^{-2} \mathrm{~s}^{-1}$ applied every $20 \mathrm{~s}$ to induce maximal fluorescence and $700 \mu \mathrm{mol}$ photons $\mathrm{m}^{-2} \mathrm{~s}^{-1}$ of actinic light to induce NPQ.

Protein extractions and immunoblotting. Cells were grown to mid-exponential phase. Forty milliliters of culture were harvested by centrifugation and lysed in a Savant FastPrep FP120 bead mill (Thermo Scientific, Karlsruhe, Germany) ${ }^{9}$. Approximately twenty-five micrograms of protein were separated in a $10 \%$ polyacrylamide gel by SDS-PAGE and blotted onto a nitrocellulose membrane. After blotting, the nitrocellulose membrane (Amersham Protran 0.1 $\mu \mathrm{m}$ NC, GE Healthcare) was cut between 35 and $40 \mathrm{kDa}$ and the top half $(40-250 \mathrm{kDa})$ was used to detect PtAureola, whereas the bottom half $(0-35 \mathrm{kDa})$ was used to detect the D1 loading control. Immunoblots to detect PtAureola were made using a custom-made antiserum specific against PtAureola (Agrisera AB, Vännas, Sweden) at a 1:1000 dilution ${ }^{9}$. The D1-specific antiserum (AS05-084, Agrisera AB) was used according to the manufacturer's instructions, at a 1:20000 dilution. Blots were developed using an Odyssey FC Imaging System (Li-Cor, Bad Homburg, Germany). An uncropped scan of the blot is shown in Supplementary Fig. 15.

\section{Data availability}

Short read sequence data have been deposited at the NCBI Sequence Read Archive (SRA) under the accession code SRP160923. The authors declare that all data supporting the findings of this study are available within the article and its Supplementary Information files or are available from the corresponding author upon request.

Received: 25 May 2018 Accepted: 31 August 2018

Published online: 25 September 2018

\section{References}

1. Falkowski, P. G., Barber, R. T. \& Smetacek, V. Biogeochemical controls and feedbacks on ocean primary production. Science 281, 200-207 (1998).

2. Pulz, O. \& Gross, W. Valuable products from biotechnology of microalgae. Appl. Microbiol. Biotechnol. 65, 635-648 (2004). 
3. Huang, W. \& Daboussi, F. Genetic and metabolic engineering in diatoms. Philos. Trans. R. Soc. Lond. B. Biol. Sci. 372, 20160411 (2017).

4. Hamilton, M. L. et al. Towards the industrial production of omega-3 long chain polyunsaturated fatty acids from a genetically modified diatom Phaeodactylum tricornutum. PLoS One 10, e0144054 (2015).

5. Kuczynska, P., Jemiola-Rzeminska, M. \& Strzalka, K. Photosynthetic pigments in diatoms. Mar. Drugs 13, 5847-5881 (2015).

6. Daboussi, F. et al. Genome engineering empowers the diatom Phaeodactylum tricornutum for biotechnology. Nat. Commun. 5, 3831 (2014).

7. Weyman, P. D. et al. Inactivation of Phaeodactylum tricornutum urease gene using transcription activator-like effector nuclease-based targeted mutagenesis. Plant. Biotechnol. J. 13, 460-470 (2014).

8. Fortunato, A. E. et al. Diatom phytochromes reveal the existence of far-redlight-based sensing in the ocean. Plant Cell 28, 616-628 (2016).

9. Serif, M., Lepetit, B., Weißert, K., Kroth, P. \& Bartulos, C. A fast and reliable strategy to generate TALEN-mediated gene knockouts in the diatom Phaeodactylum tricornutum. Algal Res. 23, 186-195 (2017).

10. Nymark, M., Sharma, A. K., Sparstad, T., Bones, A. M. \& Winge, P. A CRISPR-Cas9 system adapted for gene editing in marine algae. Sci. Rep. 6, 24951 (2016).

11. Mann, M., Serif, M., Jakob, T., Kroth, P. G. \& Wilhelm, C. PtAUREO1a and PtAUREO1b knockout mutants of the diatom Phaeodactylum tricornutum are blocked in photoacclimation to blue light. J. Plant. Physiol. 217, 44-48 (2017).

12. Slattery, S. S. et al. An expanded plasmid-based genetic toolbox enables cas 9 genome editing and stable maintenance of synthetic pathways in Phaeodactylum tricornutum. ACS Synth. Biol. 7, 328-338 (2018).

13. Daboussi, F. Advances in editing microalgae genomes. Perspect. Phycol. 4, 17-23 (2017).

14. Szczebara, F. M. et al. Total biosynthesis of hydrocortisone from a simple carbon source in yeast. Nat. Biotechnol. 21, 143-149 (2003).

15. Kim, H. \& Kim, J.-S. A guide to genome engineering with programmable nucleases. Nat. Rev. Genet. 15, 321-334 (2014).

16. Baek, K. et al. DNA-free two-gene knockout in Chlamydomonas reinhardtii via CRISPR-Cas9 ribonucleoproteins. Sci. Rep. 6, 30620 (2016).

17. Shin, S.-E. et al. CRISPR/Cas9-induced knockout and knock-in mutations in Chlamydomonas reinhardtii. Sci. Rep. 6, 27810 (2016).

18. Baek, K. et al. Photoautotrophic production of macular pigment in a Chlamydomonas reinhardtii strain generated by using DNA-free CRISPRCas9 RNP-mediated mutagenesis. Biotechnol. Bioeng. 115, 719-728 (2018).

19. Ferenczi, A., Pyott, D. E., Xipnitou, A. \& Molnar, A. Efficient targeted DNA editing and replacement in Chlamydomonas reinhardtii using Cpf1 ribonucleoproteins and single-stranded DNA. Proc. Natl Acad. Sci. USA 114, 13567-13572 (2017).

20. Sakaguchi, T., Nakajima, K. \& Matsuda, Y. Identification of the UMP synthase gene by establishment of uracil auxotrophic mutants and the phenotypic complementation system in the marine diatom Phaeodactylum tricornutum. Plant Physiol. 156, 78-89 (2011).

21. Collonnier, C. et al. CRISPR-Cas9-mediated efficient directed mutagenesis and RAD51-dependent and RAD51-independent gene targeting in the moss Physcomitrella patens. Plant. Biotechnol. J. 15, 122-131 (2017).

22. Huysman, M. J. J. et al. AUREOCHROMEla-mediated induction of the diatom-specific cyclin dsCYC2 controls the onset of cell division in diatoms (Phaeodactylum tricornutum). Plant Cell 25, 215-228 (2013).

23. Brinkman, E. K., Chen, T., Amendola, M. \& van Steensel, B. Easy quantitative assessment of genome editing by sequence trace decomposition. Nucleic Acids Res. 42, e168-e168 (2014).

24. Feri, A. et al. Analysis of repair mechanisms following an induced doublestrand break uncovers recessive deleterious alleles in the candida albicans diploid genome. MBio 7, e01109-01116 (2016).

25. Haeussler, M. et al. Evaluation of off-target and on-target scoring algorithms and integration into the guide RNA selection tool CRISPOR. Genome Biol. 17, 148 (2016)
26. Martin-Ortigosa, S. \& Wang, K. Proteolistics: a biolistic method for intracellular delivery of proteins. Transgenic. Res. 23, 743-756 (2014).

27. Gaillard, C., Moffatt, B. A., Blacker, M. \& Laloue, M. Male sterility associated with APRT deficiency in Arabidopsis thaliana results from a mutation in the gene APT1. Mol. Gen. Genet. MGG 257, 348-353 (1998).

28. Taddei, L. et al. Multisignal control of expression of the LHCX protein family in the marine diatom Phaeodactylum tricornutum. J. Exp. Bot. 67, 3939-3951 (2016).

29. Kim, S., Kim, D., Cho, S. W., Kim, J. \& Kim, J.-S. Highly efficient RNA-guided genome editing in human cells via delivery of purified Cas9 ribonucleoproteins. Genome Res. 24, 1012-1019 (2014).

30. Cermak, T. et al. Efficient design and assembly of custom TALEN and other TAL effector-based constructs for DNA targeting. Nucleic Acids Res. 39, e82 (2011).

31. Doyle, E. L. et al. TAL Effector-Nucleotide Targeter (TALE-NT) 2.0: tools for TAL effector design and target prediction. Nucleic Acids Res. 40, W117-W122 (2012).

32. Apt, K. E., Kroth-Pancic, P. G. \& Grossman, A. R. Stable nuclear transformation of the diatom Phaeodactylum tricornutum. Mol. Gen. Genet. MGG 252, 572-579 (1996).

\section{Acknowledgements}

This work was funded by a Gordon and Betty Moore Foundation grant (GBMF 4966), a Région Midi-Pyrénées grant (15058490 financial support for Accueil d'Equipes d'Excellence), an ANR JCJC grant (ANR-16-CE05-0006-01), and the 3BCAR Carnot Institute funding. We thank Dr. Fabien Nogué for stimulating scientific discussions, Dr. Peter G. Kroth for providing the PtAureola anti-serum, Dr. Jean Pierre Bouly and Dr. Angela Faciatore for discussion on NPQ analyses.

\section{Author contributions}

F.D. conceived the study. M.S., G.D., A.F., D.J. and F.D. designed the experiments. M.S., G.D., A.F., M.T., D.J. and F.D. performed and analyzed the experiments. D.J. and F.D. wrote the manuscript with support from all authors.

\section{Additional information}

Supplementary Information accompanies this paper at https://doi.org/10.1038/s41467018-06378-9.

Competing interests: The authors declare no competing interests.

Reprints and permission information is available online at http://npg.nature.com/ reprintsandpermissions/

Publisher's note: Springer Nature remains neutral with regard to jurisdictional claims in published maps and institutional affiliations.

Open Access This article is licensed under a Creative Commons Attribution 4.0 International License, which permits use, sharing, adaptation, distribution and reproduction in any medium or format, as long as you give appropriate credit to the original author(s) and the source, provide a link to the Creative Commons license, and indicate if changes were made. The images or other third party material in this article are included in the article's Creative Commons license, unless indicated otherwise in a credit line to the material. If material is not included in the article's Creative Commons license and your intended use is not permitted by statutory regulation or exceeds the permitted use, you will need to obtain permission directly from the copyright holder. To view a copy of this license, visit http://creativecommons.org/ licenses/by/4.0/.

(C) The Author(s) 2018 